DIABETES

\section{Fast-acting insulin in snail venom}

Ahorukomeye, P et al. Elife. https://doi.

org/10.7554/eLife.41574

In vertebrates, insulin is only active in its monomeric form, but the hormone tends to self-associate into stable inactive hexameres. This property means that insulin preparations for the treatment of diabetes only become effective $30 \mathrm{~min}$ after injection, when hexameric dissociation occurs. Strategies to develop faster-acting insulin by removing the region needed for oligomerization have resulted in less active products because the region is also critical for receptor activation. A type of insulin in the venom used by fish-hunting cone snails to induce hypoglycemic shock in their prey could hold the key to designing monomeric fast-acting insulin: despite lacking the region critical for self-assembly, venom insulin activated the human insulin receptor and lowered blood glucose levels in mouse and zebrafish models of diabetes.

https://doi.org/10.1038/s41684-019-0278-x

\section{DEVELOPMENTAL BIOLOGY \\ Heart regeneration in Xenopus}

Marshall, L.N. et al. PNAS 116, 3614-3623 (2019)

After injury, adult human hearts cannot compensate for the loss of damaged cardiomyocytes, owing to their limited regenerative capacity. Lost cardiomyocytes are replaced by fibrotic tissue through a remodelling process that can lead to heart failure. Zebrafish hearts, by contrast, can regenerate throughout their lifetime; researchers are trying to understand these species-specific differences to inform new therapeutic approaches for cardiac regeneration.

A new study using Xenopus laevis as a model for cardiac regeneration shows that tadpole hearts can regenerate until the larvae reach metamorphosis, which is the transition stage between the larval and the juvenile phenotype. Thyroid hormone excess or deprivation in tadpoles reduced cardiac regenerative capacity, indicating that fine-tuning of thyroid hormone availability might be necessary for successful cardiac regeneration.

\section{NEURODEGENERATIVE DISEASE}

\section{Young blood for old mice}

Das, M.M. et al. Commun. Biol. 2, 73 (2019)

Ageing induces changes in the brain that affect cognition; age-related diseases can accelerate the rate of neuronal dysfunction and lead to cognitive decline. Surgically connecting the circulatory system of a young mouse to an old mouse (heterochronic parabiosis) has been shown to counteract age-related changes in multiple organs of the old mouse; however, it remains unclear which blood components and mechanisms are responsible for this rejuvenation.

A new study reports that young bone marrow transplantation (BMT) preserved cognitive function in old recipient mice, despite irradiation-induced suppression of neurogenesis before donor cells injection. The investigators showed that young BMT preserved synaptic connections and reduced microglial activation in the hippocampus; these findings support that neurogenesis has a minimal role in the maintenance of hippocampal function-a brain region sensitive to the detrimental effects of ageing — and could have translational applications for targeting ageing- or diseaseassociated hippocampal dysfunction. $A L B$

https://doi.org/10.1038/s41684-019-0280-3

GENE THERAPY

\section{Dual gene therapy for deaf mice}

Akil, O. et al. PNAS 116, 4496-4501 (2019)

Autosomal recessive genetic forms (DFNB) account for $80 \%$ cases of profound congenital deafness. In humans, DFNB is diagnosed in the neonatal period, long after the inner ear is developed. Efforts are therefore underway to identify therapies that can reverse deafness after hearing onset. A study describes a new adeno-associated virus (AAV)-based gene therapy delivered to the mature cochlea that reversed hearing loss in mice with DFNB9, a condition caused by mutations in the OTOF gene. A dual AAV approach was used, in which the two halves of the large Otof cDNA were contained in two separate vectors. Delivery of the vectors in Otof ${ }^{-1}$ mice resulted in expression of full-length otoferlin protein via DNA recombination, and a reversal of the deafness phenotype, raising hope for patients with DFNB9.

https://doi.org/10.1038/s41684-019-0281-2

Alexandra Le Bras, Ellen P. Neff

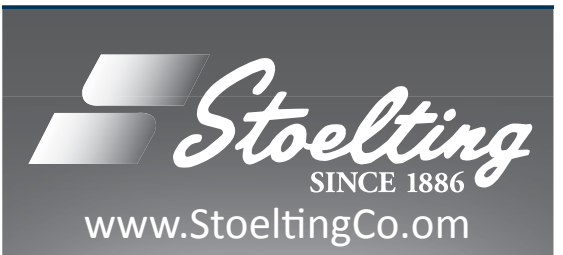

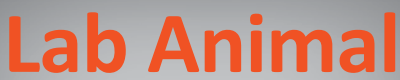
Identification

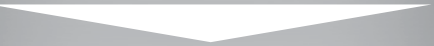

SMALL ANIMAL EARTAGS AND MARKERS
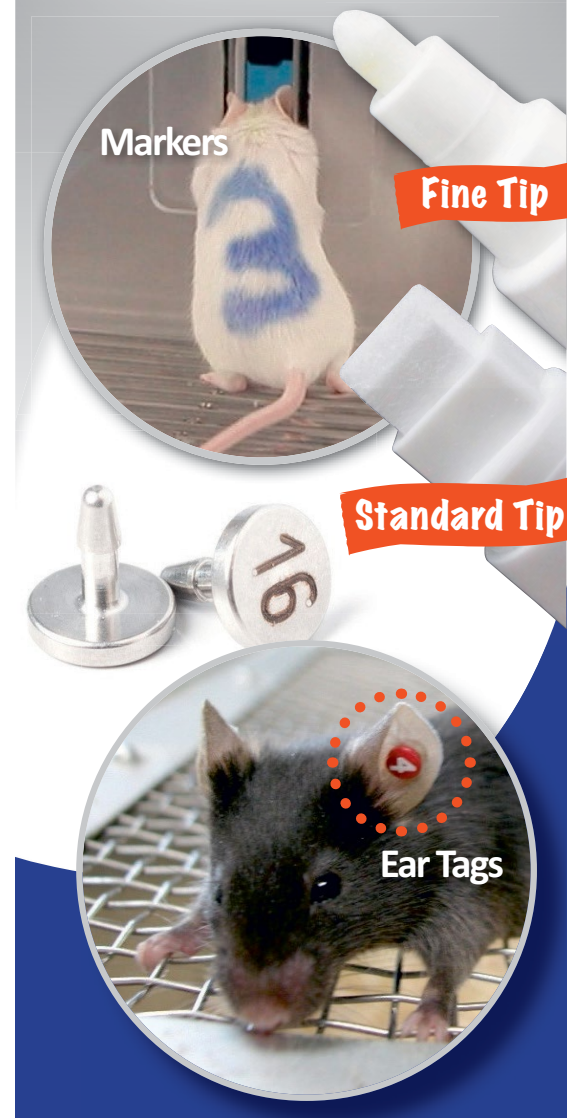

Ear Tag and Marker Features:

- Quick and easy identification

- Long-lasting

- Nontoxic, non-hazardous

- Multiple colors options available

Samples now available, Contact Stoelting for more details! info@stoeltingco.com 